\title{
A Note on the DP-Chromatic Number of Complete Bipartite Graphs
}

\author{
Jeffrey A. Mudrock*
}

August 4, 2018

\begin{abstract}
DP-coloring (also called correspondence coloring) is a generalization of list coloring recently introduced by Dvořák and Postle. Several known bounds for the list chromatic number of a graph $G, \chi_{\ell}(G)$, also hold for the DP-chromatic number of $G, \chi_{D P}(G)$. On the other hand, there are several properties of the DP-chromatic number that shows that it differs with the list chromatic number. In this note we show one such property. It is well known that $\chi_{\ell}\left(K_{k, t}\right)=k+1$ if and only if $t \geq k^{k}$. We show that $\chi_{D P}\left(K_{k, t}\right)=k+1$ if $t \geq 1+\left(k^{k} / k !\right)(\log (k !)+1)$, and we show that $\chi_{D P}\left(K_{k, t}\right)<k+1$ if $t<k^{k} / k !$.
\end{abstract}

Keywords. graph coloring, list coloring, DP-coloring.

Mathematics Subject Classification. 05C15, 05 C69.

\section{Introduction}

In this note all graphs are nonempty, finite, simple graphs unless otherwise noted. Generally speaking we follow West [14] for terminology and notation. For this note the set of natural numbers is $\mathbb{N}=\{1,2,3 \ldots\}$. The natural log function is denoted log. Given a set $A$, $\mathcal{P}(A)$ is the power set of $A$. Also, for any $k \in \mathbb{N},[k]=\{1,2,3, \ldots, k\}$. If $G$ is a graph and $S, U \subseteq V(G)$, we use $G[S]$ for the subgraph of $G$ induced by $S$, and we use $E_{G}(S, U)$ for the subset of $E(G)$ with one endpoint in $S$ and one endpoint in $U$. Also, if $v \in V(G)$ we use $N_{G}(v)$ for the set of neighbors of $v$ in $G$.

\subsection{List Coloring}

List coloring is a well known variation on the classic vertex coloring problem, and it was introduced independently by Vizing [12] and Erdös, Rubin, and Taylor [8] in the 1970's. In the classic vertex coloring problem we wish to color the vertices of a graph $G$ with as few colors as possible so that adjacent vertices receive different colors, a so-called proper coloring. The chromatic number of a graph, denoted $\chi(G)$, is the smallest $k$ such that $G$ has a proper coloring that uses $k$ colors. For list coloring, we associate a list assignment, $L$, with a graph $G$ such that each vertex $v \in V(G)$ is assigned a list of colors $L(v)$ (we say $L$

\footnotetext{
${ }^{*}$ Department of Applied Mathematics, Illinois Institute of Technology, Chicago, IL 60616. E-mail: jmudrock@hawk. iit.edu
} 
is a list assignment for $G$ ). The graph $G$ is $L$-colorable if there exists a proper coloring $f$ of $G$ such that $f(v) \in L(v)$ for each $v \in V(G)$ (we refer to $f$ as a proper L-coloring of $G$ ). A list assignment $L$ is called a $k$-assignment for $G$ if $|L(v)|=k$ for each $v \in V(G)$. The list chromatic number of a graph $G$, denoted $\chi_{\ell}(G)$, is the smallest $k$ such that $G$ is $L$-colorable whenever $L$ is a $k$-assignment for $G$. We say $G$ is $k$-choosable if $k \geq \chi_{\ell}(G)$.

It is immediately obvious that for any graph $G, \chi(G) \leq \chi_{\ell}(G)$. Erdős, Rubin, and Taylor [8] studied the equitable choosability of $K_{m, m}$ and observed that if $m=\left(\begin{array}{c}2 k-1 \\ k\end{array}\right)$, then $\chi_{\ell}\left(K_{m, m}\right)>k$. The following related result is often attributed to Vizing [12] or Erdös, Rubin, and Taylor [8], but it is best described as a folklore result.

Theorem 1. For $k \in \mathbb{N}, \chi_{\ell}\left(K_{k, t}\right)=k+1$ if and only if $t \geq k^{k}$.

We study the analogue of Theorem 1 for DP-coloring.

\subsection{DP-coloring}

Dvořák and Postle [7] introduced DP-coloring (they called it correspondence coloring) in 2015 in order to prove that every planar graph without cycles of lengths 4 to 8 is 3 -choosable. Intuitively, DP-coloring is a generalization of list coloring where each vertex in the graph still gets a list of colors but identification of which colors are different can vary from edge to edge. Following [5], we now give the formal definition. Suppose $G$ is a graph. A cover of $G$ is a pair $\mathcal{H}=(L, H)$ consisting of a graph $H$ and a function $L: V(G) \rightarrow \mathcal{P}(V(H))$ satisfying the following four requirements:

(1) the sets $\{L(u): u \in V(G)\}$ form a partition of $V(H)$;

(2) for every $u \in V(G)$, the graph $H[L(u)]$ is complete;

(3) if $E_{H}(L(u), L(v))$ is nonempty, then $u=v$ or $u v \in E(G)$;

(4) if $u v \in E(G)$, then $E_{H}(L(u), L(v))$ is a matching (the matching may be empty).

Suppose $\mathcal{H}=(L, H)$ is a cover of $G$. We say $\mathcal{H}$ is $k$-fold if $|L(u)|=k$ for each $u \in V(G)$. An $\mathcal{H}$-coloring of $G$ is an independent set in $H$ of size $|V(G)|$. It is immediately clear that $I \subseteq V(G)$ is an $\mathcal{H}$-coloring if and only if $|I \cap L(u)|=1$ for each $u \in V(G)$.

The DP-chromatic number of a graph $G, \chi_{D P}(G)$, is the smallest $k \in \mathbb{N}$ such that $G$ admits an $\mathcal{H}$-coloring for every $k$-fold cover $\mathcal{H}$ of $G$. Suppose we wish to prove $\chi_{D P}(G) \leq k$. Since every $k$-fold cover of $G$ is isomorphic to a subgraph of some $k$-fold cover, $\mathcal{H}^{\prime}=\left(L^{\prime}, H^{\prime}\right)$, of $G$ with the property that $E_{H^{\prime}}\left(L^{\prime}(u), L^{\prime}(v)\right)$ is a perfect matching whenever $u v \in E(G)$, we need only show that $G$ has an $\mathcal{H}$-coloring whenever $\mathcal{H}=(L, H)$ is a $k$-fold cover of $G$ such that $E_{H}(L(u), L(v))$ is a perfect matching for each $u v \in E(G)$.

Given a list assignment, $L$, for a graph $G$, it is easy to construct a cover $\mathcal{H}$ of $G$ such that $G$ has an $\mathcal{H}$-coloring if and only if $G$ has a proper $L$-coloring (see [5]). It follows that $\chi_{\ell}(G) \leq \chi_{D P}(G)$. This inequality may be strict since it is easy to prove that $\chi_{D P}\left(C_{n}\right)=3$ whenever $n \geq 3$, but the list chromatic number of any even cycle is 2 (see [5] and [8]).

We now briefly discuss some similarities between the DP-coloring and list coloring. First, notice that like $k$-choosability, the graph property of having DP-chromatic number at most $k$ is monotone. It is also clear that, as in the context of list coloring, if $\chi_{D P}(G)=k$, then an $\mathcal{H}$-coloring of $G$ exists whenever $\mathcal{H}$ is an $m$-fold cover of $G$ with $m \geq k$. The 
coloring number of a graph $G$, denoted $\operatorname{col}(G)$, is the smallest integer $d$ for which there exists an ordering, $v_{1}, v_{2}, \ldots, v_{n}$, of the elements in $V(G)$ such that each vertex $v_{i}$ has at most $d-1$ neighbors among $v_{1}, v_{2}, \ldots, v_{i-1}$. It is easy to prove that $\chi_{\ell}(G) \leq \chi_{D P}(G) \leq \operatorname{col}(G)$. Thomassen [13] famously proved that every planar graph is 5-choosable, and Dvořák and L. Postle [7] observed that the DP-chromatic number of every planar graph is at most 5 . Also, Molloy [11] recently improved a theorem of Johansson, and showed that every trianglefree graph $G$ with maximum degree $\Delta(G)$ satisfies $\chi_{\ell}(G) \leq(1+o(1)) \Delta(G) / \log (\Delta(G))$. Bernshteyn [4] subsequently showed that this bound also holds for the DP-chromatic number.

On the other hand, Bernshteyn [3] showed that if the average degree of a graph $G$ is $d$, then $\chi_{D P}(G)=\Omega(d / \log (d))$. This is in stark contrast to the celebrated result of Alon [1] which says $\chi_{\ell}(G)=\Omega(\log (d))$. It was also recently shown in [5] that there exist planar bipartite graphs with DP-chromatic number 4 even though the list chromatic number of any planar bipartite graph is at most 3 [2]. A famous result of Galvin [9] says that if $G$ is a bipartite multigraph and $L(G)$ is the line graph of $G$, then $\chi_{\ell}(L(G))=\chi(L(G))=\Delta(G)$. However, it is also shown in [5] that every $d$-regular graph $G$ satisfies $\chi_{D P}(L(G)) \geq d+1$.

\subsection{Outline of Results and an Open Question}

In this note we present some results on the DP-chromatic number of complete bipartite graphs. By what was mentioned in the previous subsection, we know that if $k, t \in \mathbb{N}$, $\chi_{D P}\left(K_{k, t}\right) \leq \operatorname{col}\left(K_{k, t}\right) \leq k+1$. For the remainder of this note, for each $k \in \mathbb{N}$, let $\mu(k)$ be the smallest natural number $l$ such that $\chi_{D P}\left(K_{k, l}\right)=k+1$. We have that $\mu(k)$ exists for each $k \in \mathbb{N}$ since we know by Theorem 1 ,

$$
k+1=\chi_{\ell}\left(K_{k, k^{k}}\right) \leq \chi_{D P}\left(K_{k, k^{k}}\right) \leq k+1 .
$$

This means that $\mu(k) \leq k^{k}$ for each $k \in \mathbb{N}$. The following proposition is also clear.

Proposition 2. For $k \in \mathbb{N}, \chi_{D P}\left(K_{k, t}\right)=k+1$ if and only if $t \geq \mu(k)$

Proof. If $t \geq \mu(k), k+1=\chi_{D P}\left(K_{k, \mu(k)}\right) \leq \chi_{D P}\left(K_{k, t}\right) \leq k+1$ since $K_{k, \mu(k)}$ is a subgraph of $K_{k, t}$. Conversely, if $\chi_{D P}\left(K_{k, t}\right)=k+1$, then $\mu(k) \leq t$ by the definition of $\mu(k)$.

Computing $\mu(k)$ is easy when $k=1,2$. Clearly, $\mu(1)=1$. Also, $\mu(2)=2$ follows from the fact that $\chi_{D P}\left(K_{2,1}\right) \leq \operatorname{col}\left(K_{2,1}\right)=2$, and the fact that $K_{2,2}$ is a 4-cycle which implies $\chi_{D P}\left(K_{2,2}\right)=3$. We have a tedious argument that shows $\mu(3)=6$, which for the sake of brevity, we do not present in this note. The following question lead to the discovery of both results in this note.

Question 3. For each $k \geq 4$, what is the exact value of $\mu(k)$ ?

We obtain an upper bound and lower bound on $\mu(k)$. Our first result gives us a lower bound.

Theorem 4. For $k \in \mathbb{N}$, if $t<\frac{k^{k}}{k !}$, then $\chi_{D P}\left(K_{k, t}\right)<k+1$.

Theorem 4 tells us that $\left\lceil k^{k} / k !\right\rceil \leq \mu(k)$ notice this lower bound is tight for $k=1,2$, and it is 1 away from being tight for $k=3$. We then use a simple probabilistic argument to prove our second result which gives us an upper bound on $\mu(k)$. 
Theorem 5. For $t \in \mathbb{N}$ let

$$
m=t+\left\lfloor k^{k}\left(1-\frac{k !}{k^{k}}\right)^{t}\right\rfloor
$$

Then, $\chi_{D P}\left(K_{k, m}\right)=k+1$.

Theorems 4 and 5 imply

$$
\left\lceil\frac{k^{k}}{k !}\right\rceil \leq \mu(k) \leq 1+\frac{k^{k}(\log (k !)+1)}{k !} .
$$

We suspect that $\left\lceil k^{k} / k !\right\rceil$ is closer to the exact value of $\mu(k)$ than the upper bound.

\section{Proofs of Results}

In this section we prove Theorems 4 and 5. We begin with a definition. Suppose that $\mathcal{H}=(L, H)$ is a $k$-fold cover of $G$. For any $v \in V(G)$, we say an independent set, $I$, in $H\left[\cup_{u \in N_{G}(v)} L(u)\right]$ is bad for $v$ if $|I|=\left|N_{G}(v)\right|$ and for each $w \in L(v), w$ is adjacent to some vertex in $I$. Notice that if $I$ is bad for $v$, then an $\mathcal{H}$-coloring of $G$ cannot contain $I$.

In this section we often have $G=K_{k, t}$, and we always suppose $G$ has bipartition $X=$ $\left\{v_{1}, v_{2}, \ldots, v_{k}\right\}, Y=\left\{u_{1}, u_{2}, \ldots, u_{t}\right\}$. We now mention an idea used frequently in this section. Notice that if $\mathcal{H}=(L, H)$ is a $k$-fold cover $G=K_{k, t}$, then there are precisely $k^{k}$ independent sets of size $k$ in $H\left[\cup_{v \in X} L(v)\right]$. If all of these independent sets are bad for at least one vertex in $Y$, then there is no $\mathcal{H}$-coloring of $G$. We now prove a lemma which gives us a bound on how many independent sets of size $k$ in $H\left[\cup_{v \in X} L(v)\right]$ can be bad for a vertex in $Y$.

Lemma 6. Suppose $G$ is a graph, $v \in V(G)$, and $\left|N_{G}(v)\right|=k$. Suppose that $\mathcal{H}=(L, H)$ is a $k$-fold cover of $G$. Then, there are at most $k$ ! distinct independent sets in $H\left[\cup_{u \in N_{G}(v)} L(u)\right]$ that are bad for $v$.

Proof. The result is obvious when $k=1$. So, suppose $k \geq 2$. We let $H^{\prime}=H\left[\cup_{u \in N_{G}(v)} L(u)\right]$. Suppose that $N_{G}(v)=\left\{v_{1}, v_{2}, \ldots, v_{k}\right\}$.

Let $\mathcal{C}$ denote the set of bijective functions from $[k]$ to $L(v)$. Let $\mathcal{I}$ denote the set of all independent sets in $H^{\prime}$ that are bad for $v$. We are done if $\mathcal{I}=\emptyset$, so we assume $\mathcal{I} \neq \emptyset$. We now define an injective mapping, $f: \mathcal{I} \rightarrow \mathcal{C}$. For $I \in \mathcal{I}$ suppose that $I=\left\{u_{1}, u_{2}, \ldots, u_{k}\right\}$ where $u_{i} \in L\left(v_{i}\right)$ (we know that $\left|I \cap L\left(v_{i}\right)\right|=1$ for each $i \in[k]$ ). Suppose that for each $i \in[k]$, $w_{i}$ is the one vertex in $L(v)$ to which $u_{i}$ is adjacent. Then, let $\sigma_{I}:[k] \rightarrow L(v)$ be the function defined by $\sigma_{I}(i)=w_{i}$. Since $I$ is bad for $v$, we know that $\sigma_{I} \in \mathcal{C}$. So, we can let $f(I)=\sigma_{I}$

To see that $f$ is injective, suppose that $I=\left\{u_{1}, u_{2}, \ldots, u_{k}\right\}$ and $I^{\prime}=\left\{u_{1}^{\prime}, u_{2}^{\prime}, \ldots, u_{k}^{\prime}\right\}$ are distinct elements of $\mathcal{I}$ where $u_{i}, u_{i}^{\prime} \in L\left(v_{i}\right)$ for each $i \in[k]$. This means that there must be a $j \in[k]$ such that $u_{j} \neq u_{j}^{\prime}$. Since $E_{H}\left(L(v), L\left(v_{j}\right)\right)$ is a matching, we know that $u_{j}$ and $u_{j}^{\prime}$ are adjacent to distinct vertices in $L(v)$. Thus, $f(I) \neq f\left(I^{\prime}\right)$. The fact that $f$ is injective immediately implies that $|\mathcal{I}| \leq|\mathcal{C}|=k$ !

We are now ready to prove Theorem 4 
Proof. We suppose $k \geq 3$ since the result is clear for $k=1,2$. We also assume $t \in \mathbb{N}$ since the result is clear when $t=0$. Suppose $G=K_{k, t}$.

Let $\mathcal{H}=(L, H)$ be an arbitrary $k$-fold cover of $G$. Let $H^{\prime}=H\left[\cup_{i=1}^{k} L\left(v_{i}\right)\right]$. It is clear that there are $k^{k}$ independent sets of size $k$ in $H^{\prime}$. Moreover, we know from Lemma 6 that there are at most $k$ ! independent sets in $H^{\prime}$ that are bad for $u_{j}$ for each $j \in[t]$. Since

$$
k^{k}-t(k !)>0
$$

there is an independent set, $I$ in $H^{\prime}$ such that $|I|=k$ and $I$ is not bad for any vertex in $Y$. Thus, for each $j \in[t]$, we can find a $w_{j} \in L\left(u_{j}\right)$ that is not adjacent to any vertex in $I$. Finally, $I \cup\left\{w_{1}, w_{2}, \ldots, w_{t}\right\}$ is an $\mathcal{H}$-coloring of $G$.

We now prove Theorem 5

Proof. We suppose $k \geq 2$ since the result is clear for $k=1$. Suppose $G=K_{k, t}$. We form a $k$-fold cover of $G$ by the following (partially random) process. We begin by letting $L\left(v_{i}\right)=\left\{\left(v_{i}, l\right): l \in[k]\right\}$ and $L\left(u_{j}\right)=\left\{\left(u_{j}, l\right): l \in[k]\right\}$ for each $i \in[k]$ and $j \in[t]$. Let graph $H$ have vertex set

$$
\left(\bigcup_{i=1}^{k} L\left(v_{i}\right)\right) \bigcup\left(\bigcup_{j=1}^{t} L\left(u_{j}\right)\right) \text {. }
$$

Also, draw edges in $H$ so that $H[L(v)]$ is a clique for each $v \in V(G)$. Finally, for each $i \in[k]$ and $j \in[t]$, uniformly and randomly choose a perfect matching between $L\left(v_{i}\right)$ and $L\left(u_{j}\right)$ from the $k$ ! possible perfect matchings. It is easy to see that $\mathcal{H}=(L, H)$ is a $k$-fold cover of $G$.

Note that there are exactly $k^{k}$ independent sets of size $k$ in $H\left[\cup_{i=1}^{k} L\left(v_{i}\right)\right]$. Suppose we name the $k^{k}$ functions from $[k]$ to $[k]: f_{1}, f_{2}, f_{3}, \ldots, f_{k^{k}}$. Then the $k^{k}$ independent sets of size $k$ in $H\left[\cup_{i=1}^{k} L\left(v_{i}\right)\right]$ are precisely: $I_{1}, I_{2}, \ldots, I_{k^{k}}$ where $I_{i}=\left\{\left(u_{l}, f_{i}(l)\right): l \in[k]\right\}$.

Suppose that for each $i \in\left[k^{k}\right], E_{i}$ is the event that $I_{i}$ is not bad for any vertex in $Y$. For any vertex $u \in Y$, it is easy to see that the probability that $I_{i}$ is bad for $u$ is

$$
\frac{k !((k-1) !)^{k}}{(k !)^{k}}=\frac{k !}{k^{k}}
$$

Thus, $P\left[E_{i}\right]=\left(1-k ! / k^{k}\right)^{t}$. Let $X_{i}$ be the random variable that is 1 when $E_{i}$ occurs, and it is 0 otherwise. Let $X=\sum_{i=1}^{k^{k}} X_{i}$. By linearity of expectation,

$$
E[X]=k^{k}\left(1-\frac{k !}{k^{k}}\right)^{t}
$$

Let $z=\lfloor E[X]\rfloor$. We can find a $k$-fold cover, $\mathcal{H}^{\prime}=\left(L^{\prime}, H^{\prime}\right)$, of $G$ such that at most $z$ of the independent sets of size $k$ in $H^{\prime}\left[\cup_{i=1}^{k} L^{\prime}\left(v_{i}\right)\right]$ are not bad for any vertex in $Y$. Suppose we call such independent sets: $I_{a_{1}}, I_{a_{2}}, \ldots, I_{a_{r}}$ (we know $r \leq z$ ).

Starting with $G$, we create a copy of $K_{k, t+r}$, called $M$, by adding $r$ new vertices, $w_{1}, \ldots, w_{r}$, to $Y$. We construct a $k$-fold cover of $M$ starting from $\mathcal{H}^{\prime}$ as follows. With each $w_{i}$ we associate $k$ vertices, $L^{\prime \prime}\left(w_{i}\right)$, and we add these vertices to $H^{\prime}$ along with edges so that the vertices in $L^{\prime \prime}\left(w_{i}\right)$ are pairwise adjacent. Then, for $i \in[r]$, we create a matching between $L^{\prime}\left(v_{j}\right)$ and $L^{\prime \prime}\left(w_{i}\right)$ for each $j \in[k]$ so that $I_{a_{i}}$ is bad for $w_{i}$. The result is a $k$-fold cover, $\mathcal{H}^{\prime \prime}$, of $M$ with the property that there is no $\mathcal{H}^{\prime \prime}$-coloring of $M$. Thus, $k+1=\chi_{D P}\left(K_{k, t+r}\right) \leq \chi_{D P}\left(K_{k, m}\right)$. 
Letting $t=\left\lceil k^{k} \log (k !) / k !\right\rceil$, we note

$$
t+\left\lfloor k^{k}\left(1-\frac{k !}{k^{k}}\right)^{t}\right\rfloor \leq\left\lceil\frac{k^{k} \log (k !)}{k !}\right\rceil+\frac{k^{k}}{k !} \leq 1+\frac{k^{k}(\log (k !)+1)}{k !}
$$

and the upper bound on $\mu(k)$ mentioned in the previous section follows.

Acknoledgement: The author would like to thank Anton Bernshteyn, Hemanshu Kaul, and Alexandr Kostochka for their helpful comments on this note.

\section{References}

[1] N. Alon, Degrees and choice numbers, Random Structures Algorithms 16 (2000), 364-368.

[2] N. Alon and M. Tarsi, Colorings and orientations of graphs, Combinatorica 12 (1992), 125-134.

[3] A. Bernshteyn. The asymptotic behavior of the correspondence number, Discrete Mathematics, 339 (2016), 2680-2692.

[4] A. Bernshteyn. The Johansson-Molloy Theorem for DP-coloring, arXiv: 1708.03843 (preprint), 2017.

[5] A. Bernshteyn and A. Kostochka. On differences between DP-coloring and list coloring, arXiv: 1705.04883 (preprint), 2017.

[6] A. Bernshteyn, A. Kostochka, and S. Pron, On DP-coloring of graphs and multigraphs, Siberian Mathematical Journal 58 (2017), 28-36.

[7] Z. Dvořák and L. Postle. List-coloring embedded graphs without cycles of lengths 4 to 8 , arXiv: 1508.03437 (preprint), 2015.

[8] P. Erdős, A. L. Rubin, and H. Taylor, Choosability in graphs, Congressus Numerantium 26 (1979), 125-127.

[9] F. Galvin, The list chromatic index of a bipartite multigraph, J. Combinatorial Theory Series B 63 (1995), no. 1, 153-158.

[10] A. Johansson. Asymptotic choice number for triangle free graphs. Technical Report 91-95, DIMACS, 1996.

[11] M. Molloy, The list chromatic number of graphs with small clique number, arXiv: 1701.09133 (preprint), 2017.

[12] V. G. Vizing, Coloring the vertices of a graph in prescribed colors, Diskret. Analiz. no. 29, Metody Diskret. Anal. v Teorii Kodovi Skhem 101(1976), 3-10.

[13] C. Thomassen, Every planar graph is 5-choosable, Journal of Combinatorial Theory Series B 62 (1994), 180-181.

[14] D. B. West, (2001) Introduction to Graph Theory. Upper Saddle River, NJ: Prentice Hall. 